\title{
FEASABILITY OF YEAST AND BACTERIA IDENTIFICATION USING UV-VIS-SWNIR DIFUSIVE REFLECTANCE SPECTROSCOPY
}

\author{
J. S. Silva, R. C. Martins, A. A. Vicente and J. A. Teixeira \\ $I B B$ - Institute for Biotechnology and BioEngineering, Universidade do Minho \\ Campus de Gualtar, 4710-057 Braga, Portugal
}

Keywords: Yeast, bacteria, UV-VIS-SWNIR reflectance spectroscopy, Singular value decomposition, Classification.

\begin{abstract}
UV-VIS spectroscopy is a powerfull qualitative and quantitative technique used in analytical chemistry, which gives information about electronic transitions of electrons in molecular orbitals. As in UV-VIS spectra there is no direct information on characteristic organic groups, vibrational spectroscopy (e.g. infrared) has been preferred for biological applications. In this research, we try to use state-of-the-art fiber optics probes to obtain UV-VIS-SWNIR diffusive reflectance measurements of yeasts and bacteria colonies on plate count agar in the region of 200-1200nm; in order to discriminate the following microorganisms: i) yeasts: Saccharomyces cerevisiae, Saccharomyces bayanus, Candida albicans, Yarrowia lipolytica; and ii) bacteria: Micrococcus luteus, Pseudomonas fluorescens, Escherichia coli, Bacillus cereus. Spectroscopy results show that UV-VIS-SWNIR has great potential for identifying microorganisms on plate count agar. Scattering artifacts of both colonies and plate count agar can be significantly removed using a robust mean scattering algorithm, allowing also better discriminations between the scores obtained by singular value decomposition. Hierarchical clustering analysis of UV-VIS and VIS-SWNIR decomposed spectral scores lead to the conclusion that the use of VISSWNIR light source produces higher discrimination ratios for all the studied microorganisms, presenting great potential for developing biotechnology applications.
\end{abstract}

\section{INTRODUCTION}

Spectroscopy is a powerful tool for biological applications, being applicable to liquids, solutions, pastes, powders, films, fibres, gases and surfaces, and making possible to characterize proteins, peptides, lipids, membranes, carbohydrates in pharmaceuticals, foods, plants or animal tissues (Hammes, 2005).

One of the most popular method is Infrared Spectroscopy (IR), and was firstly applied to biological materials in 1911 (Riddle et al., 1956). In the 1950s and 1960s research IR spectroscopy began to be applied for microorganism differentiation, but this research was abandoned due to the unsatisfactory results obtained with dispersive spectrometers (Dziuba et al., 2007). These were ignored during 20 years, until modern interferometric Fourier Transform InfraRed spectrometers (FT-IR) and statistical computing methodologies became available (Dziuba et al., 2007).

Recent techniques using FT-IR allowed microbiological characterization and the discrimination at level of sorting better species and strains. Attenuated total reflection and IR micro-spectroscopy have been associated to the discrimination and identification of strains according to taxonomic classification, gram $+/$ - factor, or even susceptibility to antibiotics and grown medium (Mariey et al., 2001).

FT-IR has also been used to identify lactic acid bacteria strains (e.g. Lactobacillus, Lactococcus, Leuconostoc, Pediococcus and Streptococcus (Dziuba et al., 2007), and the rapid identification of Acinetobacter species (Winder et al., 2004). An extensive FT-IR spectroscopy database for the identification of bacteria from the two suborders Micrococcineae and Corynebacterineae (Actinomycetales, Actinobacteria) as well as other morphologically similar genera was established in 2002 by Helene Oberreuter and its team (Oberreuter et al., 2002). Furthemore, FTIR was used for the first time to determine the ratios of different yeast species (Saccharomyces cerevisiae, Hanseniaspora uvarum) and two yoghurt lactic acid bacteria (Lactobacillus acidophilus, Streptococcus salivarius ssp. thermophilus) in suspensions 
Table 1: Studied microorganisms characteristics and experimental conditions: Gram factor, colony colour and shape and integration time.

\begin{tabular}{lcccccc}
\hline & & & & \multicolumn{2}{c}{ Integration Time (ms) } \\
Microorganism & Gram & Colony color & Shape & Medium & UV-VIS (ms) & VIS-NIR (ms) \\
\hline Saccharomyces cerevisiae & na & white & spherical & YPD & 70 & 66 \\
Saccharomyces bayanus & na & white & spherical & YPD & 45 & 61 \\
Candida albicans & na & white & spherical & YPD & 70 & 66 \\
Yarrowia lipolytica & na & white & rod & YPD & 70 & 61 \\
Micrococcus luteus & - & yellow & spherical & TSA & 20 & 19 \\
Pseudomonas fluorescens & - & translucent & rod & MP & 20 & 30 \\
Escherichia coli & - & translucent & rod & LB & 30 & 13 \\
Bacillus cereus & + & opaque & rod & LB & 36 & 61 \\
\hline
\end{tabular}

of distilled water (Oberreuter et al., 2000).

Raman Spectroscopy has also shown great potential for microorganisms identifications in microscopy, such as for Candida yeast strains and bacterial strains such as Staphylococus, Enterococus and Echerichia Coli) (Guibeta et al., ). Applications are also found in oral hygiene, for the identification of Streptococcus mutants, S. sanguis, S.intermedius and S. oralis (Berger and Zhu, 2003). Moreover, this technique is currently used to identify baterials cells of Staphylococcus under different cultivation conditions (Harz et al., 2005) and single yeast cells (Rch et al., 2005). Fluorescence Spectroscopy (FS) is one of the most important spectroscopic techniques in molecular biology, and consequently can also be used to microbiological identification. FS applications can be found on the differentiation of yeast and bacteria, by their intrinsic fluorescence to UV excitation (Bhatta et al., 2005).

UV-VIS-SWNIR spectroscopy is one of the most widely used techniques in analytical chemistry, but it has almost not been used for microorganism identification. This is perhaps attributed to the fact that UV-VIS spectroscopy records transmitions between electron energy levels from molecular orbitals, instead of vibrational or structural oscillation of molecular groups as in the infrared region. It is widely accepted that vibrational spectroscopy is more adequate for organic chemistry measurement than transitional spectroscopy. Nevertheless an asset of this technique has never been done use for microbiological identification.

Electronic transitions in the UV-VIS region depend upon the energy involved. For any molecular bound (sharing a pair of electrons), orbitals are a mixture of two contributing orbitals $\sigma$ and $\pi$, with corresponding anti-bounding orbitals $\sigma^{*}$ and $\pi^{*}$, respectively. Some chemical bounds present characteristic orbital conditions, ordered by higher to lower order energy transitions: i) alkanes ( $\left.\sigma \rightarrow \sigma^{*} ; 150 \mathrm{~nm}\right)$; ii) carbonyls ( $\sigma \rightarrow \pi^{*}$; 170nm); iii) unsaturated com- pounds ( $\pi \rightarrow \pi^{*}$; 180nm); iv) molecular bounds to $\mathrm{O}, \mathrm{N}, \mathrm{S}$ and halogens $\left(n \rightarrow \sigma^{*} ; 190 \mathrm{~nm}\right)$; and v) carbonyls ( $\left.n \rightarrow \pi^{*} ; 300 \mathrm{~nm}\right)$. As most UV-VIS spectrometers yield a minimum wavelength of $200 \mathrm{~nm}$, this technique has been considered to provide lower information in terms of functional groups when compared to IR, being the spectral differences mostly attributed to conjugated $\pi \rightarrow \pi^{*}$ transitions and $n \rightarrow \pi^{*}$ transitions (Perkauparus et al., 1994).

Only recording $\pi \rightarrow \pi^{*}$ and $n \rightarrow \pi^{*}$ transitions above the $200 \mathrm{~nm}$ is however not totally a handicap. Many organic molecules present conjugated unsaturated and carbonyls bounds, such as aminoacids, phospholipids, free fatty acids, phenols and flavonoids, peroxides, peptides and proteins, sugars and their polymers absorbance in these bands. Furthermore, many biological molecules present chromophore groups, which increase the absortion in the UV-VIS region, such as: nitro, nitroso, azo, azo-amino, azoxy, carbonyl and thiocarbonyl, which can be used to identify microorganisms.

UV-VIS-SWNIR has some advantages to FTIR for microbiological identification in plate count agar. The lower wavelength turns this radiation attractive due to the lower penetration, being easier to monitor surfaces than NIR or MIR radiation. Furthermore, state-of-the-art fiber-optics miniature UV-VISSWNIR are today affordable for mobile applications such as identification of microorganisms in surfaces, using spectroscopy may became feasible in a near future. Although UV-VIS retrieves only molecular spectroscopy information, today's equipments also include high frequency vibrational spectroscopy in the SWNIR region, giving important information on water, fats and proteins which may be used to discriminate between microorganisms.

In this research was tried to discriminate both Yeasts and Bacteria of comonly used in microbiology laboratories: i) yeasts: Saccharomyces cerevisiae, Saccharomyces bayanus, Candida albicans, Yarrowia lipolytica, ii) bacteria: Micrococcus luteus, 


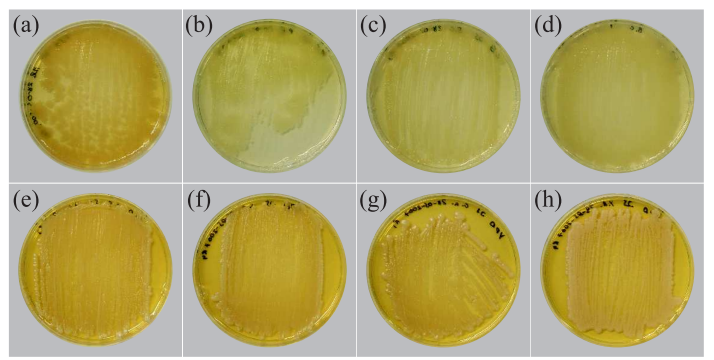

Figure 1: Yeast and bacteria growth media: (a) Tryptic Soy Agar (TSA): Micrococcus luteus; (b) Pseudomonas Isolation Agar (MP) (Pseudomonas fluorescens); (c) LuriaBertani (LB): Escherichia coli, (d) LB: Bacillus cereus; (e) YPD (Saccharomyces cerevisiae); (f) YPD (Saccharomyces bayanus); (g) YPD (Candida albicans); and (h) YPD (Yarrowia lipolytica).

Pseudomonas fluorescens, Escherichia coli, Bacillus cereus; under plate count agar growth media.

The physical properties of the UV-VIS-SWNIR spectra can also provide a great potential for the identification of microorganism, using multivariate statistical analysis and signal processing techniques. Microbes may not be directly identified by their main colony chemical composition but rather by characteristic metabolites produced under different growth media. This is especially true for yeasts that exhibit one of the most complex metabolisms in this study. Therefore, not only the colony but changes in the composition of the plate count agar in the surroundings of each colony are expected to affect the UV-VIS-SWNIR spectra in order to obtain significant discrimination between the different microorganisms spectra. Therefore, the main objective of this research work were to investigate the discrimination potential of UV-VIS and VIS-NIR wavelengths to classify the following microorganisms: i) yeasts: Saccharomyces cerevisiae, Saccharomyces bayanus, Candida albicans, Yarrowia lipolytica, ii) bacteria: Micrococcus luteus, Pseudomonas fluorescens, Escherichia coli, Bacillus cereus; under plate count agar growth media.

\section{MATERIALS AND METHODS}

\subsection{Sample Preparation}

The microorganisms were obtained from the microbiological collection of the IBB - Institute for Biotechnology and Bioengineering at the University of Minho. The microorganisms were incubated under aerobic conditions at $35^{\circ} \mathrm{C}$ during $72 \mathrm{~h}$. Micrococcus luteus was cultivated on Difco Tryptic Soy Agar (TSA) while Pseudomonas fluorescens was grown on
Difco Pseudomonas Isolation Agar (MP). Escherichia coli and Bacillus cereus were cultivated on Difco Luria-Bertani Agar (LB). Yeast strains were grown on Difco YPD Agar (YPD), at the same temperature and time (Difco, 2005).

\subsection{Spectroscopy}

Yeast and bacteria UV-VIS-SWNIR spectroscopy analysis was performed using the fiber optic spectrometer AvaSpec-2048-4-DT (2048 pixel, 200$1100 \mathrm{~nm})$. Standart reflection UV-VIS and VISSWNIR probes, models FCR-7UV200-2ME and FCR-7IR200-2-ME (Avantes, 2007). A xenon and halogen light sources, models AvaLight XE-2000 and AvaLight-Hal were used for UV-VIS and VIRSWNIR transmission measurements respectively; and recorded using AvaSoft 6.0 (Avantes, 2007). Transmission measurements were performed at the room temperature of $18 \pm 2^{\circ} C$, and: (a) UV-VIS: the xenon lamp was let to stabilize during $20 \mathrm{~min}$; (b) VIS-NIR: the tungsten lamp lamp was let to stabilize during 15 min. The dark spectra was recorded and measurements were taken with linear and electric dark correction. Both light spectra were monitored by statistically assessing the reproducibility of the light source with measurements of light during the several days of the experiment. Fifteen spectra replicates were recorded of UV-VIS and VIS-SWNIR measurement of both plate count agar and microorganisms colonies to study scattering effects. Futhermore, spectra were obtained inside a box designed to isolate the environmental light and maintain the probe at $90^{\circ}$ angle with the plate agar.

\subsection{Spectral Analysis}

\subsubsection{Spectra Pre-processing}

Table 1 presents the UV-VIS and VIS-NIR spectra acquisition conditions. Experimental setup has shown that it is impossible to use the same integration time for the different microorganisms. Under these circumstances, all the collected spectra were normalized $\mathbf{x}_{\text {norm }}$ to remove this effect:

$$
\mathbf{x}_{\text {norm } i}=D_{S} \times \frac{\mathbf{x}_{\text {raw } i}}{\max \left(\mathbf{x}_{i}\right)}
$$

where $\mathbf{x}_{\text {raw } i}$ is the original spectra, $D_{S}$ is the detector saturation value (14000 counts) and $\mathbf{x}_{\text {norm } i}$ the $\mathrm{i}$ 'th spectra normalized by its maximum value and resized to the detector saturation.

Furthermore, as most plaque count agar growth media are translucid, the signal recorded is in majority the media information. To increase spectral vari- 

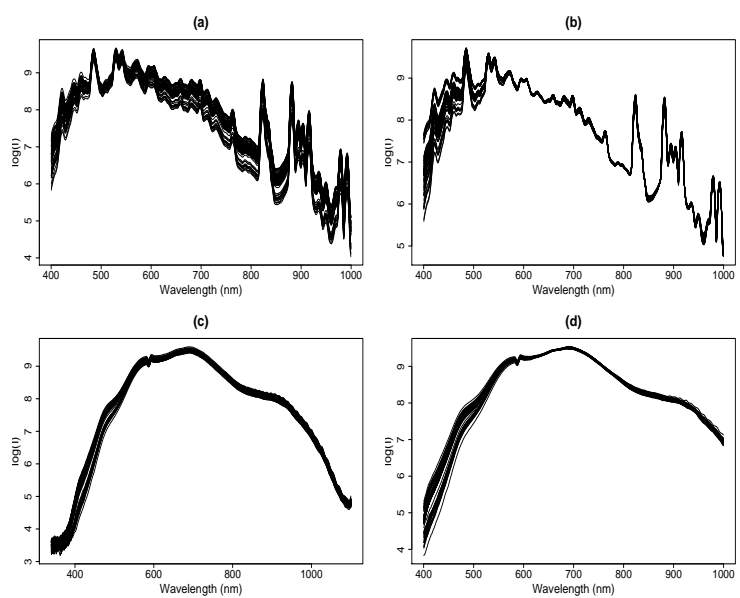

Figure 2: Plaque count agar spectra: (a) raw UV-VIS; (b) MSC UV-VIS; (c) raw VIS-NIR; (d) MSC VIS-NIR.

ance, the normalized media spectra matrix was subtracted to the microorganisms spectra, obtaining the spectra matrix $(\mathbf{x})$, which is thereafter subjected to robust mean scattering correction, and singular value decomposition.

\subsubsection{Robust Mean Scattering Correction}

The collected spectra were smoothed by using a Savisky-Golay filter (length $=4$, Order= 2) (?) prior to any exploratory data analysis procedure. Afterwards, the spectra was pre-processed using a modified multiplicative scatter correction algorithm (Gallagher et al., 2005; Martens and Stark, 1991; Martens et al., 2003). Each spectra is corrected by using the following equation:

$$
\mathbf{x}_{c o r r}=\mathbf{x} b+a=\mathbf{x}_{r e f}
$$

The $a$ and $b$ are computed by minimizing the following error:

$$
\mathbf{e}_{j}=b \mathbf{x}_{j}+a-\mathbf{x}_{r e f}
$$

where the $\mathbf{x}_{j}$ is the $j$ sample spectra and $\mathbf{x}_{r e f}$ is a reference spectra.

This RMSC algorithm is based on the application of the robust least squares method to determine the $a$ and $b$ matrices ensuring that spectral areas that do not correspond to scattering artifacts are not taken into account. The robust least squares algorithm is implemented by the re-weighted least squares with the weights computed by using the Huber function. The algorithm high breakdown point (50\%) means that existent outliers will not distort the model fitting (eq. 3) and thus, the $a$ and $b$ scatter correction parameters are determined using only consistent spectral areas. The iterative algorithm can be described, briefly as follow: 1) set the reference spectra $\left(\mathbf{x}_{r e f}\right)$ equal to the sample
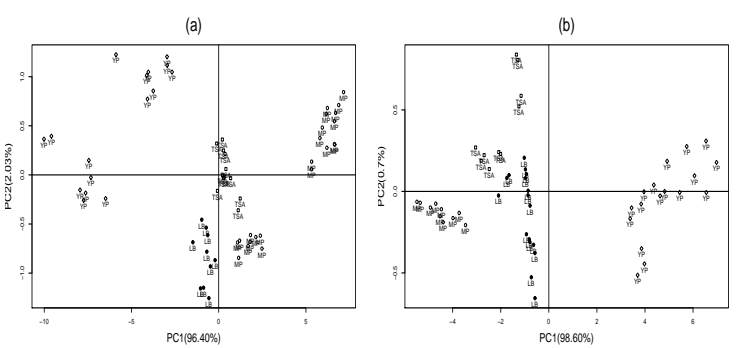

Figure 3: Growth media Gabriel plot: (a) UV-VIS (PC1 $(96.40 \%)$, PC2 $(2.3 \%)$ and (b) VIS-NIR (PC1 (98.60\%), PC2 $(0.70 \%)$ to the corresponding growth media: TSA $(\square)$, MP (॰), LB $(\bullet)$ and YPD $(\diamond)$.

spectra closest to the median spectra; 2) correct the remaining sample spectra by applying the above described robust least squares procedure; eq.3) recompute the median spectra and iterate until convergence.

\subsubsection{Singular Value Decompostion}

Singular value decomposition (SVD) is a blind signal technique widely used in spectroscopy data, where the corrected spectra $\left(\mathbf{x}_{\text {corr }}\right)$ is decomposed in order of magnitude of variation directions in the variable space (wavelengths). Generally, most variability is captured in the first principal components (PC), where as, in good signal to noise spectral data, noise is captured in the last orthogonal decompositions, and therefore a spectra can be decomposed as:

$$
\mathbf{x}_{\text {corr }}=\widehat{\mathbf{x}}+\varepsilon(\mathbf{x})
$$

where the $\widehat{\mathbf{x}}$ is the signal and $\varepsilon(\mathbf{x})$ is the estimated noise of $\mathbf{x}$. Spectra matrix $\mathbf{x}_{\text {corr }}$ can be decomposed by (SVD), where:

$$
\mathbf{x}=\mathbf{U S V}^{\mathbf{T}}
$$

where US are the scores, $\mathbf{V}^{\mathbf{T}}$ the loadings and the S singular values, respectively (Jolliffe, 1986; Krzanowski, 1998; Baig and Rehman, 2006).

To distinguish between the number of relevant decompositions, one can determine the number of relevant singular values by performing $\mathrm{n}$ randomizations of the original spectra matrix (x) (Manly, 1998). In this research, 5000 randomizations were performed by rotating the spectral scope value at the same wavelengths among the different samples, in order to not violate the spectral continuity. Singular values from the original spectra $\mathbf{x}$ above the 1st singular value of randomized spectra $\left(\mathbf{x}_{\text {rand }}\right)$ define the number of independent singular values of the original signal where is possible to discriminate the different microorganisms spectra:

$$
\widehat{\mathbf{x}}=\mathbf{U} \mathbf{S}_{r e l v} \mathbf{V}^{\mathbf{T}}{ }_{r e l v}
$$



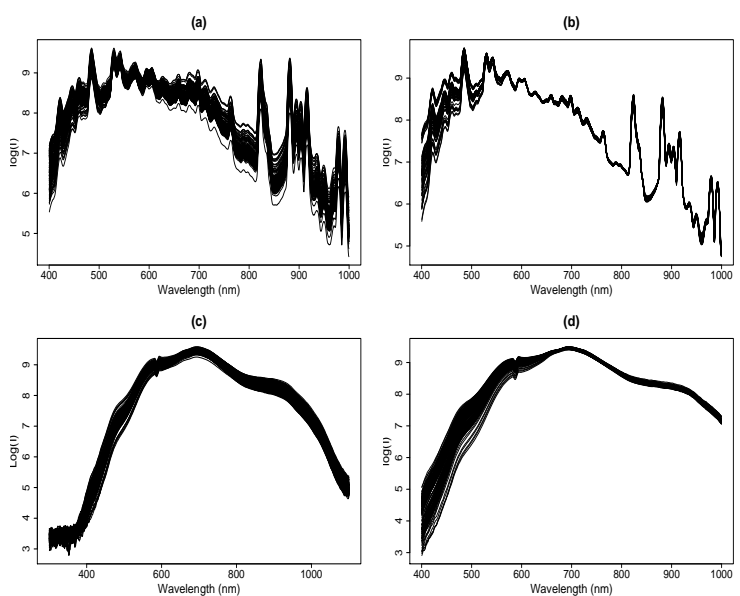

Figure 4: Microorganisms spectra: (a) raw UV-VIS; (b) MSC UV-VIS; (c) raw VIS-NIR; (d) MSC VIS-NIR.

Where $\mathbf{U} \mathbf{S}_{\text {relv }}$ and $\mathbf{V}^{\mathbf{T}}$ relv are the statistically relevant scores and loading of $\mathbf{x}$, respectively. To further discriminate between the microorganisms spectra, the relevant PC's scores $\left(\mathbf{U S}_{\text {relv }}\right)$ were subjected to hierarchical clustering according to the euclidean distance, to determine the potential of using UV-VIS and VISNIR to identify the studied yeast and bacteria. All statistical computing analysis were performed using R (R-Project, 2006).

\section{RESULTS AND DISCUSSION}

\subsection{Spectral Analysis}

Figure 2 presents the UV-VIS and VIS-NIR plate count agar growth media spectra, respectively. It is possible to observe in Figure 2(a) and 2(c) that all plate count agar are highly dispersive, generating a high scattering effect. This undesired scattering effect is due to the light path length to be very sensitive to the probe angle, particles in the agar, surface texture of both agar and petri disk. If the light scattering effect is not corrected, variance due to this physical phenomenon affects significantly the chemical interpretation of the spectra due to scattering artifacts.

As pure additive effects of light scattering are rarely observed in samples with complex compositions, being mainly of multiplicative origin, the growth media spectra was subjected to RMSC, being the corrected spectra presented in Figures 2 (b) and 2 (c), respectively. By directly comparing Figures 2 (a)-(b), and 2 (c)-(d), one can observe the scattering effect is obtained in both light sources. After applying the RMSC one can observe that, this scattering artifacts are significantly reduced in the region of 700-1000nm, but nevertheless both light sources present higher degree of spectral variance in the region of $400-700 \mathrm{~nm}$.

Furthermore, it is observable that all spectra are proportional to each other. Variation is mostly in terms of signal intensity than in spectra shape. In this sense it is difficult to distinguish the different growth media by direct spectra comparison. Figure 3 presents the Gabriel plot (PC1 vs PC2) of the growth media spectra, for UV-VIS and VIS-NIR wavelengths, respectively. Both UV-VIS and VIS-NIR biplots evidence optical properties of the growth media, being thin mostly described by the 1 st principal component. In both biplots, it is possible to observe that the MP growth media is the most translucid and YPD the most opaque, and therefore PC1 can be interpreted as the amount of signal that the detector records. Media such as TSA and LB present similar spectra records. Such is mainly attributed to their similar composition in terms of main components such as sodium chloride, agar and water.

Results show that MP and YPD media are more suceptible to variability than TSA and LB in spectroscopy terms. As no chemical assessment was performed to the media, we cannot present the cause for this source of variation.

Figure 4 presents the UV-VIS and VIS-NIR microorganisms spectra, respectively. Similarly to the growth media, the microbe spectra exhibits high scattering artifacts (see Figures $4 \mathrm{a}$ and $4 \mathrm{c}$ ). The scattering effect is in this case due to the light scattering at the colony surface and growth media, which significantly affects the observed spectra. Similarly to the growth media, scattering is significantly high in both light sources and evenly distributed from 450 to 1000 $\mathrm{nm}$, nevetheless scattering is significantly reduced by the RMSC algorithm. The corrected spectra presents higher variability in the region of $400-700 \mathrm{~nm}$, but nevertheless it is difficult to recognize directly spectral characteristics that distinguish the different microorganisms under study, and therefore SVD analysis is necessary.

\subsection{Singular Value Decomposition Analysis}

Figure 5 presents the Gabriel plot of the first two components obtained by SVD (PC1(78.40\%), PC2 $(8.03 \%)$ ), and the corresponding hierarchical clustering analysis. The first two decompositions ensure the majority of the spectral variation to proportional to the average spectrum ( $\mathrm{PC1}(78.40 \%)$ ), and that linear variance also with discriminant power $(8.03 \%)$, evidences smaller but discriminate spectral 
(a)

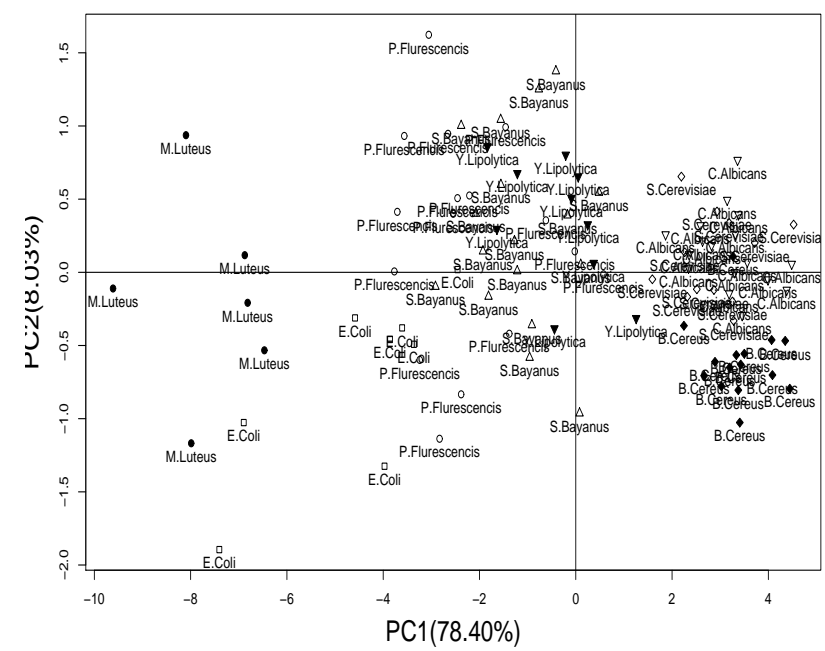

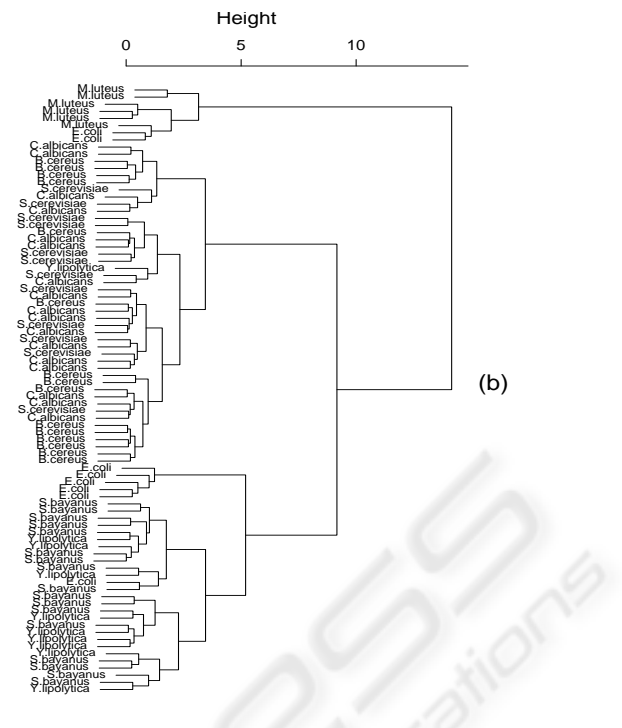

Figure 5: UV-VIS Microorganisms Spectra PCA Analysis: (a) Gabriel Plot (PC1 (78.40\%), PC2 (8.03\%); S. cerevisiae ( $\diamond), S$. bayanus $(\triangle)$, C. albicans $(\nabla)$, Y. lipolytica $(\nabla)$, M. luteus $(\bullet)$, P. fluorescens $(\circ)$, E. coli $(\square)$ and B. cereus $(\diamond)$; (b) hierarquical clustering of Microorganisms.

differences between the studied microorganisms.

PC1 $(78.40 \%)$ discriminates between spectral intensity, being possible to observe that colonies of B. cereus, C. albicans and S.cerevisiae, present the higher scores indicating that the colonies of this microorganisms are well suited for diffusive reflectance. Although the relative proximity in the scores space, it is observable that $B$. cereus, $C$. albicans and $S$. cerevisiae are discriminated by the 2 nd PC. In the group, it is possible to observe a higher similarity between $C$. albicans and S.cerevisiae spectra than with B. Cereus.

It is further observable that although $S$. bayanus and Y. lipolityca exhibit similar spectral intensity, their spectra is possible to he discriminated in the 2nd PC. S. bayanus who presents larger variability then $Y$. lipolityca, being more difficult to identify.

E. coli and M. luteus colonies are distinguishable from all the other microorganisms group. These present the lowest signal intensity, and the proximity of the two spectra may in part he due to the growth media spectral similarity between TSA and LB, as show in Figure 3a. Nevertheless, the UV-VIS spectra decomposition is capable of discriminating between E. coli and M. luteus spectra.

In the UV-VIS, P. fluorescens spectra has proved to he highly unreproducible, being its scores well spread throughout the 2nd PC. This unreproducibility is attributed to the high translucent of both P. fluorescens colonies and the MP growth media, and to the experimental microbiological technique. As microorganisms were inoculated using a inoculating loop, a significant part of radiation is dif- fused into de media in P. fluorescens. Better growth may in the future improve the spectral measurement and therefore its identification, as already observed in E. coli colonies, which although are smaller, are capable of developing thicker colonies.

Dispite the experimental difficulties hierarchical clustering analysis presented in Figure 5b shows that the majority of the studied microorganisms cluster together with exception of $P$. fluorescens. This gives good perspectives of using UV-VIS spectroscopy for microorganisms identification in plate count agar after experimental and signal processing improvements.

The VIS-SWNIR spectra also exhibits high scattering artifacts in the 400-1000nm region (see Figure $4 \mathrm{c}$ ), the scattering effect is effectively removed by the RMSC algorithm used during the spectral preprocessing. Figure $4 d$, shows that in the corrected spectra, variance is higher in the $400-700 \mathrm{~nm}$ as already observed as with the UV-VIS light source. The majority of the spectra are proportional to each other, varying only in signal intensity.

Figure 6a presents the Gabriel plot of the first two PCs of the decompose VIS-NIR spectra (PC1 $(75.40 \%)$, PC2 $(10.95 \%)$, and the corresponding scores hierarchical clustering. Similarly to the UVVIS spectra, the first spectra decomposition discriminating variance proportional to the average spectra, and PC2 (lower variance) tends to discriminate smaller details between the spectra of the studied microorganisms, evidencing that VIS-NIR is a viable methodology for identification microorganisms on plate count agar. PC1 (75.40\%) of VIS-SWNIR spec- 
(a)

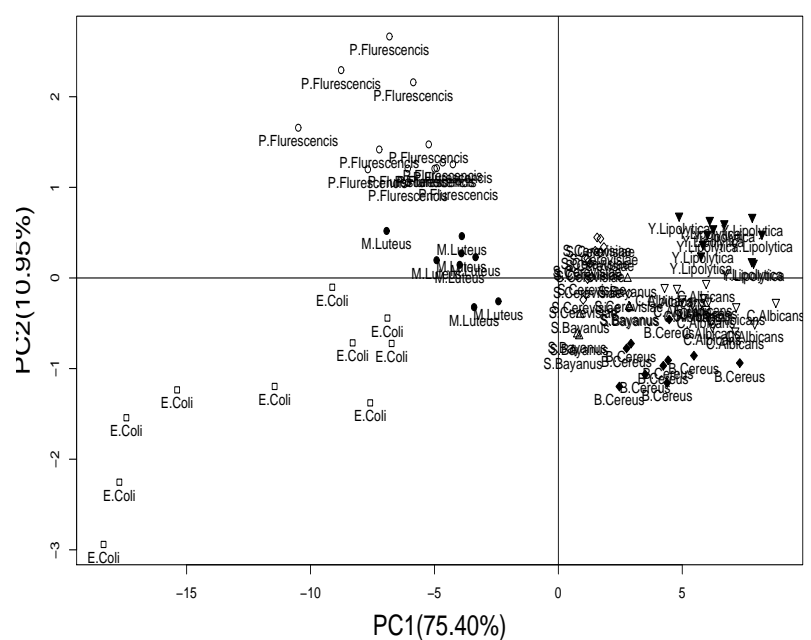

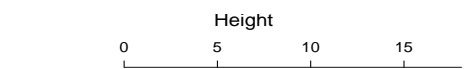

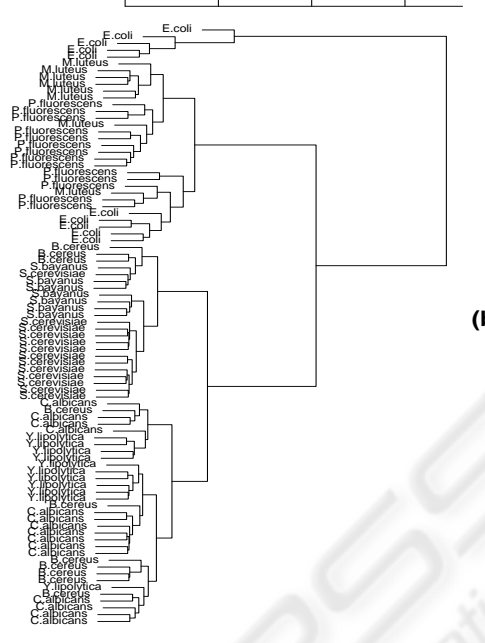

(b)

Figure 6: VIS-NIR Microorganisms SpectraPCA Analysis: (a) Gabriel Plot (PC1 (75.40\%), PC2 (10.95\%); S. cerevisiae. $(\diamond), S$. bayanus $(\triangle)$, C. albicans $(\nabla)$, Y. lipolytica $(\nabla)$, M. luteus $(\bullet)$, P. fluorescens $(\circ)$, E. coli $(\square)$ and B. cereus $(\diamond) ;(b)$ hierarquical clustering of Microorganisms.

tra presents a clear discrimination between groups of microorganisms: (a) B. cereus, S.cerevisiae, $S$. bayanus, Y. lipolityca, C. albicans, and (b) M. luteus, E. coli and P. fluorescens. PC2 (10.95\%) is capable of discriminate the microorganisms inside these two groups, and therefore, complete discrimination is achievable with VIS-SWNIR wavelengths.

In the VIS-SWNIR spectra E. coli and P. fluorescens present higher dispersion of signal when compared to the rest of the spectra of microorganisms. Nevertheless, results reproductivity inside these two groups is significantly higher than with the UV-VIS measurements; indicating that translucid colonies were better identified under the VIS-SWNIR radiation. Furthermore, data suggest that VIS-SWNIR may be better to be used under non-optimal measuring conditions. This is especially problematic if we want to identify microorganisms in plate count agar with similar compositions and metabolism.

\subsection{Methodology Improvements}

This preliminary study shows that UV-VIS and VISSWNIR reflection spectroscopy has a great potential for rapid qualitative discrimination of yeasts and bacteria in plate count agar. Nevertheless, both experimental methodology and signal processing techniques should be improved to take advantage of the information contained in the UV-VIS-SWNIR.

Improvements to the experimental methodology are necessary to improve discrimination in the studied region of the spectra. For example, the use of liquid cultures to replicate microorganisms and then innoc- ulate by a droplet on the surface agar will allow the growth of compact and thicker colonies. Small and thin colonies lead to readings with much media information, because the light is reflected from both sites of the agar. This is particularly relevant for E. coli and $P$. fluorescens because of the small size of the colonies and small thickness of $P$. fluorescens; being the spectra highly affected by the media composition, leading to sistematic errors in discrimination. Microorganisms signal spectra can also be maximised by reducing the growth media thickness. Such minimises light dispersion accross the agar, and increases the colony spectral intensity by passing through it the reflected light; ans as well by optimising fiber optics diffusive reflectance position and angle control, minimizing scattering effects in the colonies and growth media.

Scattering artifacts and small noise were successfully removed by pre-processing the spectra with the RMSC and Savisky-Golay filter, respectively; being possible to achieve high-quality and resolution final spectra before signal treatment. Improvements can also be performed to the spectra processing procedure. Methods such as the combination of logistic partial least squares (log-PLS) with multiblock (e.g. UV-Vis+Vis-Nir spectra) can provide a new insight to the discrimination of microorganisms using spectroscopy. If these methodologies provide good discriminations, more robust techniques such as the use of wavelets for compressing the original spectra and orthogonal-PLS classification of spectra will be tested. 


\section{CONCLUSIONS}

Results show that UV-VIS-SWNIR spectroscopy is a feasable technology for plate count agar microorganisms identifications. The robust mean scattering correction algorithm was able to efficiently remove the growth media and colonies scattering artifacts, allowing a better interpretation of the singular value decomposition scores loading. In this exploratory experiment, VIS-SWNIR wavelengths were able to produce better discriminations between microorganisms than the UV-VIS region. Nevertheless, experimental methodology and signal processing improvements proposed may increase the discrimination resolution, making UV-VIS-SWNIR an attractive methodology for rapid microorganisms identification in plate count agar.

\section{REFERENCES}

Avantes (2007). Avaspec-2048-4-dt/-rm.
http://www.avantes.com.

Baig, S. and Rehman, F. (2006). Signal modeling using singular value decomposition. In Advances in Computer, Information, and Systems Sciences, and Engineering. Springer Netherlands.

Berger, A. and Zhu, Q. (2003). Identification of oral bacteria by raman microspectroscopy. In Journal of Modern Optics 50(15-17): 2375-2380. Taylor and Francis Group.

Bhatta, H., Goldys, E., and Learmonth, R. (2005). Rapid identification of microorganisms by intrinsicfluorescence. In Imaging, Manipulation, and Analysis of Biomolecules and Cells: Fundamentals and Applications III, . SPIE.

Difco, L. (2005). Difco manual : dehydrated culture media and reagents for microbiology. - VII. Difco Laboratories, Detroit, USA.

Dziuba, B., Babuchowski, A., Naleczb, D., and Niklewicz, M. (2007). Identification of lactic acid bacteria using ftir spectroscopy and cluster analysis. In International Dairy Journal 17: 183189. Elsevier.

Gallagher, N. B., Blake, T., and Gassman, P. (2005). Application of extended inverse scattering correction to mid-infrared reflectance of soil. In Journal of Chemometrics 19: 271-281.

Guibeta, F., Marieya, L., Pichonb, P., Traverta, J., Denisb, C., and Amiela, C. Discrimination and classification of enterococci by fourier transform infrared spectroscopy.

Hammes, G. G. (2005). Fundamentals of spectroscopy. In Spectroscopy for the Biological Sciences. John Wiley Sons, Inc.

Harz, M., Rosch, P., Peschke, K.-D., Ronneberger, O., Burkhardtb, H., and Popp, J. (2005). Micro-raman spectroscopic identification of bacterial cells of the genus staphylococcus and dependence on their cultivation conditions. In Analyst 130: 15431550. The Royal Society of Chemistry.

Jolliffe, I. (1986). Principal Component Analysis. Springer, New York, USA.

Krzanowski, W. J. (1998). Principles of Multivariate Data Analysis. Oxford University Press, Oxford, UK.

Manly, B. F. (1998). Randomization, Bootstrap and Monte Carlo Methods in Biology. Chapman and Hall, London, UK, 2nd edition.

Mariey, L., Signolle, J., Amiel, C., and Travert, J. (2001). Discrimination, classification, identification of microorganisms using ftir spectroscopy and chemometrics. In Vibrational Spectroscopy 26: 151-159. Elsevier

Martens, H., Nielsen, J. P., and Engelsen, S. B. (2003). Light scattering and light absorbance separated by extended multiplicative signal correction. application to near-infrared transmission analysis of powder mixtures. In Analytical Chemistry 75(9): 394-404. American Chemical Society.

Martens, H. and Stark, E. (1991). Extended multiplicative signal correction and spectral interference subtraction: new preprocessing methods for near infrared spectroscopy. In Journal of Pharmaceutical and Biomedical Analysis 9: 625-635. American Chemical Society.

Oberreuter, H., Mertens, F., Seiler, H., and Scherer, S. (2000). Quantification of micro-organisms in binary mixed populations by fourier transform infrared (ftir) spectroscopy. In Letters in Applied Microbiology 2000, 30: 8589. The Society for Applied Microbiology.

Oberreuter, H., Seiler, H., and Scherer, S. (2002). Identification of coryneform bacteria and related taxa by fourier-transform infrared (ft-ir) spectroscopy. In International Journal of Systematic and Evolutionary Microbiology 52: 91100. IUMS.

Perkauparus, H., Grinter, H., and Therfall, T. (1994). Uv-Vis spectroscopy and its applications. Springer-Verlag, New York, USA.

R-Project (2006). R: A programming environment for data analysis and graphics. URL: http://www.r-project.org.

Rch, P., Harz, M., Schmitt, M., and Popp, J. (2005). Raman spectroscopic identification of single yeast cells. In $J$. Raman Spectrosc 36: 377379. Wiley InterScience.

Riddle, J., Kabler, P., Kenner, B., Bordner, R., Rockwood, S., and Stevenson, H. (1956). Bacterial Identification by Infrared Spectrophotometry. Cincinnati, Ohio.

Winder, C., Carr, E., Goodacre, R., and Seviour, R. (2004). The rapid identification of acinetobacter species using fourier transform infrared spectroscopy. In Journal of Applied Microbiology 96, 328339. The Society for Applied Microbiology. 